OPEN

SUBJECT AREAS:

DISEASES

NEURODEVELOPMENTAL DISORDERS

Received

2 December 2013

Accepted

9 April 2014

Published

2 May 2014

Correspondence and requests for materials should be addressed to P.Z. (pierluigi. zoccolotti@uniromal.

\title{
Failure to learn a new spatial format in children with developmental dyslexia
}

\author{
Maria Pontillo ${ }^{1,2}$, Maria De Luca ${ }^{1}$, Andrew W. Ellis ${ }^{3}$, Chiara Valeria Marinelli ${ }^{1}$, Donatella Spinelli ${ }^{1,4}$ \\ \& Pierluigi Zoccolotti ${ }^{1,2}$
}

'Neuropsychology Unit, IRCCS Fondazione Santa Lucia, via Ardeatina 306, 00179 Rome, Italy, ${ }^{2}$ Psychology Department, Sapienza University of Rome, via dei Marsi, 78, 00185 Rome, Italy, ${ }^{3}$ Department of Psychology, University of York, York, YO10 5DD, United Kingdom, "Department of Human Movement, Social and Health Sciences, University of Rome "Foro Italico", piazza Lauro De Bosis 15, 00135 Rome, Italy.

A general problem in studying children with developmental dyslexia is how to separate inefficiency in learning on the one hand from exposure to written texts on the other. To evaluate dyslexic children's learning abilities with graphemic materials, we tested their improvement in a condition that minimized previous experience with words (i.e., "novel words") and with the standard, horizontal spatial letter array (i.e., a non-canonical "zigzag" format). We selected five pairs of children with dyslexia and (younger) typically developing readers matched for reading speed and accuracy in these specific conditions. Reading performance on novel words in the zigzag format was measured in 23 sessions; learning curves were fitted by power functions. Similar to typically developing readers, children with dyslexia improved their reading of novel words presented in the new format; however, their rate of learning was slower than that of typically developing readers. Furthermore, their learning to read in the new format did not generalize to novel untrained items, whereas significant generalization was present in typically developing readers. As the failure to generalize learning of the spatial format could not be attributed to reduced experience, it indicates a genuine disability and points to impaired perceptual learning as a factor in developmental dyslexia.

hildren with developmental dyslexia fail to learn to read effectively. This learning disability prevents the development of accurate and fluent reading despite adequate school attendance, normal intelligence and absence of gross neurological disorders, and has widespread effects on their lives, with important negative consequences on academic achievement and self-esteem (e.g. $\left.{ }^{1}\right)$. A general problem in studying these children is to discriminate between inefficiency in learning on the one hand and exposure to written texts on the other; in fact, because of their pervasive reading disturbance, children with dyslexia are much less willing to spend time reading than normal readers ${ }^{2}$. Consequently, they have less exposure to written texts than their peers.

In the present study, we aimed to evaluate dyslexic children's learning abilities in a condition that minimized their previous experience with texts by considering two aspects of this experience: $i$ ) the reading materials and $i$ ) the spatial array of letters in reading. As for reading materials, we used novel words (i.e., legal strings of letters without any corresponding entry in the lexicon), so that neither children with dyslexia nor typically developing readers had previous experience with them. As for spatial array, we used a non-canonical zigzag format which was unfamiliar to both groups (see an example in the Methods section). This letter arrangement should minimize the difference between groups in one of the perceptual abilities supporting reading, that is extracting visual information from a horizontal string of letters.

In framing our study we refer to a recent influential model, the Local Combination Detector (LCD) model ${ }^{3}$, which has already being used to describe the effect of word degradation ${ }^{4}$. According to this model, written words are encoded by a hierarchy of detectors tuned to increasingly larger and more complex word fragments (visual features, single letters, bigrams, quadrigrams and, possibly, words). At the neural level, bilateral information from letter features and single letter converges to the left occipital-temporal region called visual word form area (VWFA); here, a posterior-to-anterior gradient is present with a progression in selectivity to increasingly word-like stimuli ${ }^{4-6}$. Thus, over years of practice, frequent combinations of letters are selected to be represented by dedicated neurons $s^{4}$, and the VWFA becomes attuned to the regularities of the writing system, yielding fast parallel processing in reading ${ }^{4,6}$. Notably for the present study, this perceptual learning is selective for standard print, i.e. words aligned horizontally and printed at high contrast in a standard font. Studies in typically developing readers have shown that, when presentation deviates greatly from this hyper-learned format, reading speed decreases and word length effects emerge, indicating a shift from parallel to effortful serial processing. This occurs 
Table 1 | Description of participants. Some characteristics of dyslexic and typically developing readers are reported. The children with dyslexia were below their age norms for reading speed and accuracy as assessed by a standard ltalian reading test ${ }^{39,40}$. The control readers performed near average (i.e., approximately 0 in terms of $z$ score depending on individual grade level) on the same test and were younger than the children with dyslexia by about three and a half years. All children demonstrated normal intelligence on Raven's Coloured Matrices

\begin{tabular}{|c|c|c|c|c|c|c|}
\hline Children with dyslexia & Age & Sex & Grade & Raven test & Reading speed & Reading accuracy \\
\hline $\begin{array}{l}1 \mathrm{~d} \\
2 \mathrm{~d} \\
3 \mathrm{~d} \\
4 \mathrm{~d} \\
5 \mathrm{~d} \\
\text { Average } \\
\text { SD }\end{array}$ & $\begin{array}{r}13.5 \\
13.2 \\
13.5 \\
13.3 \\
14.0 \\
\mathbf{1 3 . 5} \\
\mathbf{0 . 3}\end{array}$ & $\begin{array}{c}F \\
M \\
M \\
F \\
M\end{array}$ & $\begin{array}{l}7 \\
7 \\
7 \\
7 \\
8\end{array}$ & $\begin{array}{r}26 \\
32 \\
26 \\
29 \\
31 \\
\mathbf{2 8 . 8} \\
\mathbf{2 . 8}\end{array}$ & $\begin{array}{r}-0.32 \\
-0.88 \\
-2.26 \\
-2.13 \\
-4.11 \\
-\mathbf{1 . 9 4} \\
\mathbf{1 . 4 7}\end{array}$ & $\begin{array}{r}-3.41 \\
-2.39 \\
-3.41 \\
-7.61 \\
-6.90 \\
-\mathbf{4 . 7 4} \\
\mathbf{2 . 3 5}\end{array}$ \\
\hline Typically developing readers & Age & Sex & Grade & Raven test & Reading speed & Reading accuracy \\
\hline $\begin{array}{l}1 \mathrm{c} \\
2 \mathrm{c} \\
3 \mathrm{c} \\
4 \mathrm{c} \\
5 \mathrm{c} \\
\text { Average } \\
\text { SD }\end{array}$ & $\begin{array}{r}10.0 \\
9.5 \\
10.8 \\
10.7 \\
8.5 \\
9.9 \\
0.9\end{array}$ & $\begin{array}{c}F \\
M \\
M \\
M \\
F\end{array}$ & $\begin{array}{l}4 \\
4 \\
5 \\
5 \\
3\end{array}$ & $\begin{array}{l}28 \\
27 \\
28 \\
27 \\
27 \\
\mathbf{2 7 . 4} \\
\mathbf{0 . 5}\end{array}$ & $\begin{array}{l}1.05 \\
0.66 \\
0.49 \\
0.20 \\
0.07 \\
\mathbf{0 . 5 0} \\
\mathbf{0 . 3 9}\end{array}$ & $\begin{array}{r}0.53 \\
-0.10 \\
0.03 \\
0.19 \\
0.02 \\
\mathbf{0 . 1 3} \\
\mathbf{0 . 2 4}\end{array}$ \\
\hline
\end{tabular}

with low-contrast ${ }^{7}$, rotated $^{8}$, or highly spaced ${ }^{4,9}$ words as well as with words presented in case-alternation ${ }^{10,11}$, zigzag or vertical mode ${ }^{12}$. Results of neuroimaging, ERP and MEG studies ${ }^{4,13,14}$ indicate that reading degraded words activates dorsal posterior parietal areas, pointing to the role of attentional areas in reading tasks. In proficient readers, this effortful serial processing emerges most clearly when the automatic and parallel processing supported by the VWFA cannot deal with stimuli, as in the case of degraded words, since these stimuli are out of the limits of the perceptual expertise of the system ${ }^{4}$.

Notably, slow, serial processing dominate the reading of children with dyslexia also with standard texts (as indicated by the presence of large length effects ${ }^{15}$ ) as if they were unable to optimize the functioning of their VWFA. Indeed, there is now considerable evidence indicating that children with dyslexia show very poor activation of the VWFA in various reading tasks (e.g., for a review ${ }^{16}$ ). However, it is unclear whether this is the cause or the effect of their learning disturbance. As mentioned above it is difficult to separate inefficiency of learning on the one hand from exposure to written texts on the other. In the present learning study, we controlled experience with orthographic material using novel words rather than real words. We also controlled experience with format using a zigzag display of letters rather than a standard horizontal array. The zigzag format was selected because it proved quite disruptive in preliminary testing and decreased reading speed more than other format manipulations, such as vertical presentation or case alternation. According to the LCD model $^{3}$, the zigzag presentation should leave intact the visual analysis of single letters performed bilaterally, and the first stage of VWFA processing, but should impact on later stages, starting from the level where bigrams are computed. In fact, zigzag presentation activates non-aligned detectors, and this spatial arrangement is out of perceptual expertise of VWFA ${ }^{4}$. Thus, the contribution of effortful serial processing, with attentional selection of space-distributed letters, should be important in carrying out the task.

A case series of children with dyslexia and typically developing readers participated in the present study (see Table 1). We selected pairs of dyslexic and (younger) typically developing readers matched for reading speed (vocal reaction times, RT) and accuracy on our experimental task (i.e., reading zigzag novel words) before training. Starting the training from these comparable baselines allows addressing two key issues in dyslexia. First, are dyslexic children able to learn to read novel words in a new zigzag format at the same rate as typically developing readers? To test this, we examined the perform- ance of the children's pairs through a sufficiently large number of sessions to reliably estimate their curve of learning (based on the law of practice hypothesis that learning occurs following a power func$\left.\operatorname{tion}^{17}\right)$. Second, if children with dyslexia are able to learn through training, is this learning specific to the trained items or does it extend to new untrained items? Generalization was tested by presenting "new", untrained novel words in the same zigzag format at the end of the training. The performance on these items was compared to that on the original set of stimuli at the pre- and post-training. Some decay is expected when comparing performance on these new items with the items subjected to training. However, better performance on the new novel words with respect to pre-training (when zigzag novel words were presented for the first time) would indicate that children learnt to read in this non-canonical format (i.e., generalization of format). By contrast, a large performance decay with the new untrained items (with respect to post-training) equaling the performance at the pre-training, would indicate specific learning for trained items and no generalization of format.

\section{Results}

Learning curves based on RTs are presented in Fig. 1, separately for the two groups of readers (individual curves are presented in Supplementary Information files). Due to the selection criterion, children with dyslexia and typically developing readers had closely comparable baselines in the pre-training session 1 (see details in Table 2 top and bottom for RT and accuracy data, respectively). To allow for a full appreciation of the effect of training, on the right of Fig. 1 (bar plot), the performance on the new set of untrained novel words at the post-training session is also presented.

During training, typically developing readers improved at a fast rate, closely following a power function of practice, both in terms of RTs (Fig. 1) and errors (Fig. 2) ${ }^{18}$; details of the fitting functions are presented in the captions of the two figures. Dyslexics' RTs and accuracy also improved progressively with training (again well fit by a power function). However, their rate of learning was slower than that of typically developing readers (as indicated by a lower power function coefficient). Thus, by the end of training they were slower and more inaccurate than typically developing readers (see Table 2, POST: trained NW). At the post-training session with the new set of novel words (black bar in Fig. 1), the performance of children with dyslexia was very similar to that at the first session; by contrast, typically developing readers (white bar in Fig. 1) showed 
- Children with dyslexia
-- - Typically developing readers

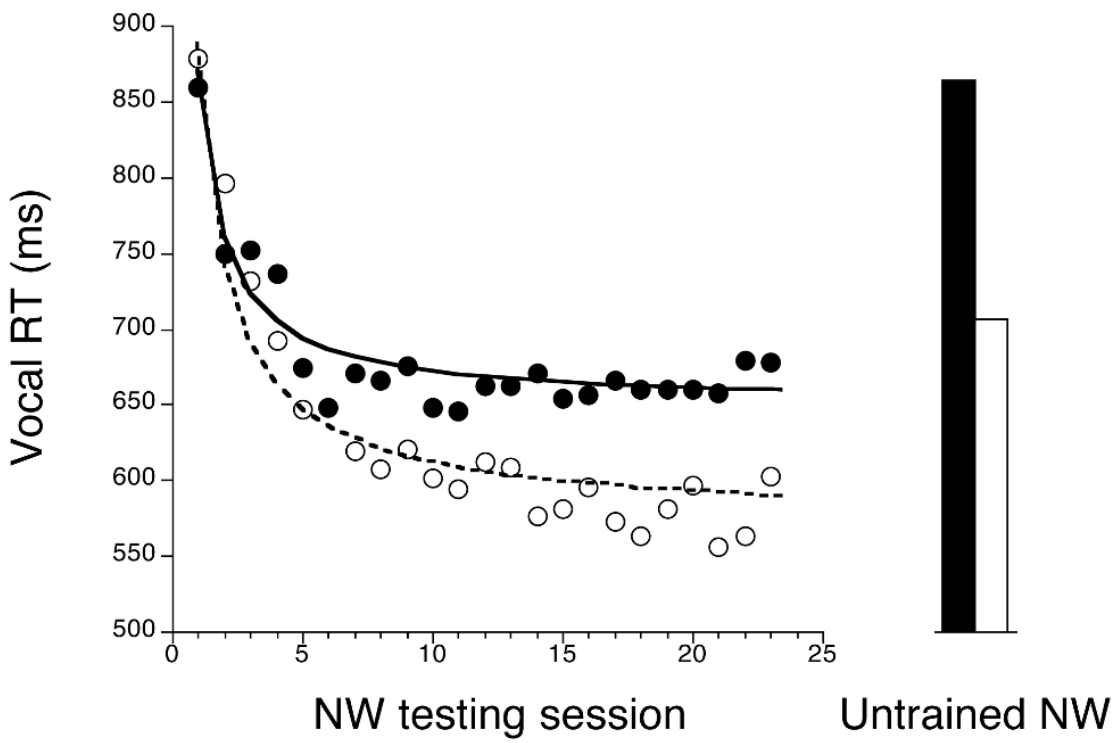

Figure $1 \mid$ RTs for zigzag novel words (NW) as a function of the number of testing sessions. Open symbols and white bars report the averages for controls; filled symbols and dark bars report data for dyslexic children. RT data were fit by power functions separately for the groups of dyslexic children (continuous lines) and controls (dotted lines). The learning curves were well fit by a power function for both groups of children $\left(\mathrm{R}^{2}=0.92\right.$ and $\mathrm{R}^{2}=0.88$ for control and dyslexic children, respectively). The equations describing the learning curves were $y=220 x^{-1.00}+650(2)$ and $y=318 x^{-.88}+570(3)$, for dyslexic children and controls, respectively. The bars represent RTs for untrained novel words at the post-training session.

an improvement in performance with respect to the beginning of training.

The RT data were formally analyzed by linear mixed effect model analyses (summarized in Table 3; for description of the models see the Methods section).

The effect of "training" was examined in the two groups by comparing pre vs post RTs in reading zigzag novel words (model $A$, see Linear mixed effect model analyses section). The analysis showed the significance of the training effect $(F(1,813.7)=298.5, P<0.0001)$ as well as the group $\times$ training effect $(F(1,805.6)=20.2, P<0.0001)$. Both groups of children showed a significant training effect (children with dyslexia: pre $=859.2 \mathrm{~ms}$, post $=677.2 ; P<0.001$; typically developing readers: pre $=878.6 \mathrm{~ms}$; post $=603.0 \mathrm{~ms} ; P<0.001$ ), but the effect was numerically larger in typically developing readers (diff. $275.6 \mathrm{~ms}$ ) with respect to children with dyslexia (diff. $182.0 \mathrm{~ms}$ )(see Tables 2 and 3). Due to the pairing selection, groups did not differ in the pre-training condition but differed in the posttraining condition $(P<0.001)$. With regard to the random effects, the effect of item was significant ( Wald $Z=2.7, P<0.01$ ) but the effect of participant was not ( Wald $Z=1.4$, n.s.). This indicates that individual differences in the two case series of children did not appreciably contribute to generating the observed effects.

The effect of "session" was examined by comparing RTs in each of the 23 sessions with the same novel words (model $B$, Linear mixed effect model analyses section). The analysis showed the significance of the effect of group $(F(1,9925.8)=456.4, P<0.0001)$, session $(F$ $(22,9925.3)=61.5, P<0.0001)$ and group $\times \operatorname{session}(F(22,9925.2)$ $=9.4, P<0.0001)$. An exploration of the interaction shows that the groups differed significantly from the third session onwards; no difference was present for the first presentation of the zigzag novel words and a tendency $(P=0.067)$ was present for the second presentation. Again, the random effect of item was significant (Wald $Z$ $=4.7 ; P<0.0001$ ), but the effect of participant was not (Wald $Z=$ 1.4, n.s.).
The effect of "stimulus material" was examined by comparing RTs to trained novel words at post-training with RTs to "new" untrained novel words (see model $C$, Linear mixed effect model analyses section). The analysis showed the significance of the effect of group ( $F$ $(1,784.9)=108.9, P<0.0001)$, stimulus material $(F(1,786.0)=$ $171.7, P<0.0001)$ and group $\times$ stimulus material $(F(1,785.1)=7.8$, $P<0.01)$. The interaction showed that both groups of children were slower on untrained than trained targets (children with dyslexia: untrained $=863.2 \mathrm{~ms}$, trained $=677.2 ; P<0.0001$; typically developing readers: untrained $=706.2 \mathrm{~ms}$; trained $=603.0 \mathrm{~ms} ; P<$ 0.0001 ); however, the interaction showed that the difference in reading trained with respect to untrained novel words was larger for children with dyslexia than typically developing readers (children with dyslexia: diff. $=186.0 \mathrm{~ms}$; typically developing readers: diff. $=103.2 \mathrm{~ms}$; see Table 2). Also in this case, the effect of item was significant (Wald $Z=3.1, P<0.01$ ), but the random effect of participants was not (Wald $Z=1.4$, n.s.).

The "generalization of format" to untrained novel words was examined comparing RTs in pre-training to RTs to untrained novel words after training (model $D$, Linear mixed effect model analyses section). The analysis showed the significance of the effect of group $(F(1,707.6)=11.0, P<0.001)$, generalization of format $(F(1,717.4)$ $=22.3, P<0.0001)$ and group $\times$ generalization of format $(F(1$, $702.4)=31.7, P<0.0001)$. The interaction indicates that typically developing readers showed faster RTs to untrained novel words in the post-training compared to the pre-training condition $(P<0.001$; in Fig. 1, compare the white bar with the open circle in session 1; see also individual and group data in Table 2 top), whereas children with dyslexia did not (in Fig. 1, compare the dark bar with the filled circle in session 1; see also Table 2 top). The random effect of item was significant ( Wald $Z=2.3, \mathrm{P}<0.05$ ), but the effect of participant was not (Wald $Z=1.4$, n.s.).

In Table 4, we detail (at both individual and group levels) how much of the improvement with training could be ascribed to item- 
Table 2 | Median vocal RTs (top) and accuracy (bottom) for zigzag novel words (NW). Each child with dyslexia was individually matched with a typically developing reader on the basis of his/her performance on both vocal RTs and percentage of errors in reading zigzag novel words at the pre-training session (baseline). Individual dyslexic-control pairs of readers are presented in each row. The "PRE" column represents the baseline performance in the first session with the 50 zigzag novel words. The "POST: trained NW" column reports performance after 22 sessions with the same list. The "POST: untrained NW" column reports performance on a new list of 50 novel words (matched for bigram frequency and initial phoneme with the trained list). Individual and averaged group data are reported separately for children with dyslexia and typically developing readers

\begin{tabular}{|c|c|c|c|c|c|c|c|}
\hline $\begin{array}{l}\text { Children with } \\
\text { dyslexia }\end{array}$ & PRE: Baseline & $\begin{array}{l}\text { POST: trained } \\
\text { NW }\end{array}$ & $\begin{array}{l}\text { POST: } \\
\text { untrained NW }\end{array}$ & $\begin{array}{c}\text { Typically } \\
\text { developing readers }\end{array}$ & PRE: baseline & POST: trained NW & $\begin{array}{l}\text { POST: } \\
\text { untrained NW }\end{array}$ \\
\hline \multicolumn{8}{|l|}{ Vocal RTs (ms) } \\
\hline $\begin{array}{l}M S \\
D B \\
A D \\
V G \\
M A \\
\text { Average } \\
\text { SD }\end{array}$ & $\begin{array}{c}661 \\
722 \\
840 \\
901 \\
1172 \\
\mathbf{8 5 9 . 2} \\
\mathbf{1 9 8 . 9}\end{array}$ & $\begin{array}{l}591 \\
567 \\
751 \\
641 \\
836 \\
\mathbf{6 7 7 . 2} \\
\mathbf{1 1 3 . 5}\end{array}$ & $\begin{array}{c}875 \\
674 \\
865 \\
712 \\
1190 \\
\mathbf{8 6 3 . 2} \\
\mathbf{2 0 3 . 4}\end{array}$ & $\begin{array}{c}\text { AR } \\
\text { MA } \\
\text { GI } \\
\text { IA } \\
\text { LI } \\
\text { Average } \\
\text { SD }\end{array}$ & $\begin{array}{c}715 \\
715 \\
741 \\
1097 \\
1125 \\
\mathbf{8 7 8 . 6} \\
\mathbf{2 1 2 . 8}\end{array}$ & $\begin{array}{l}520 \\
590 \\
653 \\
499 \\
753 \\
\mathbf{6 0 3 . 0} \\
\mathbf{1 0 3 . 5}\end{array}$ & $\begin{array}{l}600 \\
672 \\
788 \\
540 \\
931 \\
\mathbf{7 0 6 . 2} \\
\mathbf{1 5 6 . 1}\end{array}$ \\
\hline \multicolumn{8}{|c|}{ Accuracy (percentage of errors) } \\
\hline $\begin{array}{l}M S \\
D B \\
A D \\
V G \\
M A \\
\text { Average } \\
\text { SD }\end{array}$ & $\begin{array}{l}22 \\
12 \\
14 \\
16 \\
24 \\
\mathbf{1 7 . 6} \\
\mathbf{5 . 2}\end{array}$ & $\begin{array}{l}0 \\
4 \\
2 \\
4 \\
4 \\
\mathbf{2 . 8} \\
\mathbf{1 . 8}\end{array}$ & $\begin{array}{l}24 \\
32 \\
12 \\
32 \\
20 \\
\mathbf{2 4 . 0} \\
\mathbf{8 . 5}\end{array}$ & $\begin{array}{c}\text { AR } \\
\text { MA } \\
\text { GI } \\
\text { IA } \\
\text { LI } \\
\text { Average } \\
\text { SD }\end{array}$ & $\begin{array}{c}14 \\
22 \\
16 \\
6 \\
20 \\
\mathbf{1 5 . 6} \\
\mathbf{6 . 2}\end{array}$ & $\begin{array}{l}0 \\
2 \\
2 \\
0 \\
4 \\
1.6 \\
1.7\end{array}$ & $\begin{array}{c}12 \\
20 \\
8 \\
26 \\
30 \\
\mathbf{1 9 . 2} \\
\mathbf{9 . 2}\end{array}$ \\
\hline
\end{tabular}

specific learning vs generalization of format. In controls, improvement on trained items $(275.6 \mathrm{~ms})$ was due to learning the format $(172.4 \mathrm{~ms})$ and the specific items (103.2 ms). By contrast, there was no improvement in the ability of dyslexic children to generalize learning of the zigzag format to untrained novel words; that is, the improvement after training (182.0 ms pre-post training difference) was entirely due (100\%) to learning specific items in the zigzag format $(186.0 \mathrm{~ms})$, not the format per se $(-4.0 \mathrm{~ms})$.

\section{Discussion}

Through repeated presentation of novel words in a novel format, both typically developing readers and children with dyslexia progres-

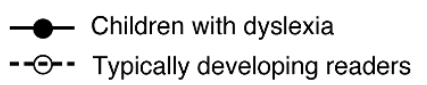

Children with dyslexia

Typically developing readers

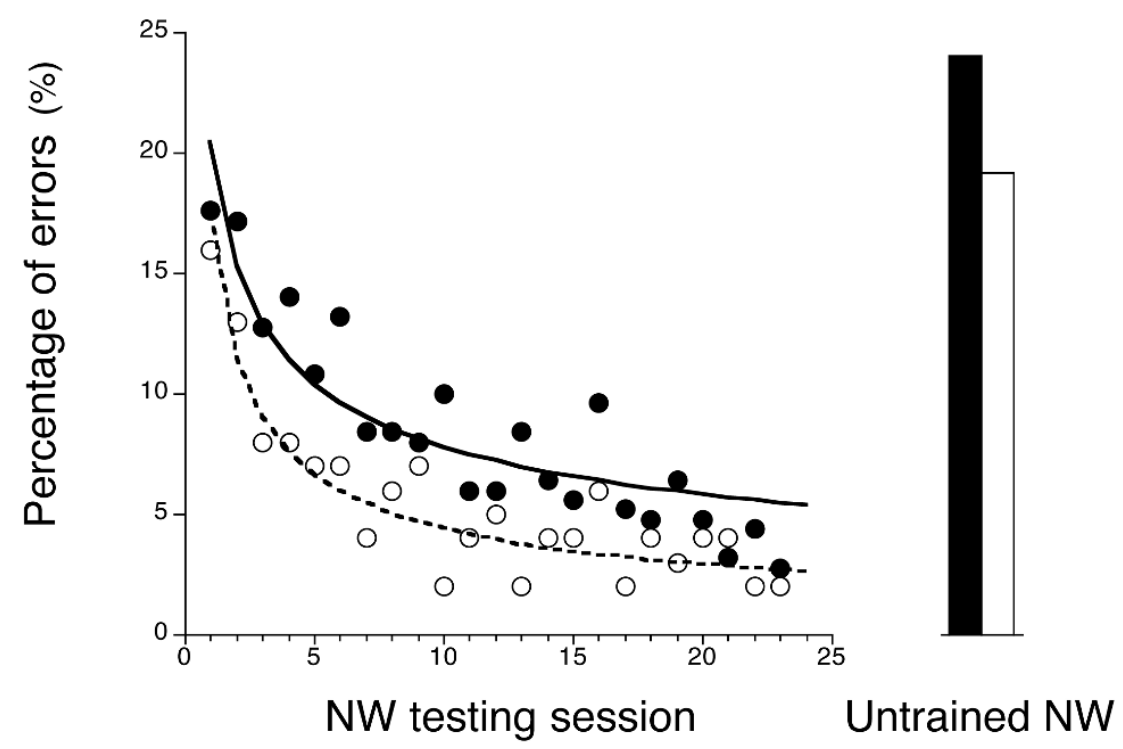

Figure $2 \mid$ Accuracy data (percentage of errors) for zigzag novel words (NW) as a function of testing sessions, separately for the two groups of children. Filled circles: dyslexic children; open circles: controls. Accuracy data were fit by power functions separately for the groups of dyslexic children (continuous lines) and controls (dotted lines). The equations describing the learning curves were $y=20.5 x^{-.42}+0(4)$ and $y=17.5 x^{-0.60}+0$ (5), for dyslexic children and controls, respectively. The bars represent accuracy for untrained novel words at the post-training session. Note that also for accuracy learning by training was higher in controls than dyslexic children; however, generalization to untrained items was low for both groups. 


\begin{tabular}{|c|c|c|c|c|}
\hline MODEL & EFFECT & Degrees of freedom & $\mathrm{F}$ & Probability level \\
\hline \multirow{2}{*}{ zigzag trained novel words } & Training & $1,813.7$ & 298.48 & 0.000 \\
\hline & Group $\times$ Training & $1,805.6$ & 20.18 & 0.000 \\
\hline $\begin{array}{l}\text { B) Session: RTs at each of the } 23 \\
\text { presentations with the trained non-words }\end{array}$ & Group & $1,9925.8$ & 456.40 & 0.000 \\
\hline C) Stimulus material: post-training RTs on & Group & $1,784.9$ & 108.87 & 0.000 \\
\hline trained novel words vs RTs on new & Stimulus material & $1,786.0$ & 171.68 & 0.000 \\
\hline untrained novel words & Group $\times$ Stimulus material & $1,785.1$ & 7.77 & 0.005 \\
\hline D) Generalization of format: RTs in pre- & Group & $1,707.6$ & 11.04 & 0.001 \\
\hline training vs RTs to untrained novel words & Generalization of format & $1,717.4$ & 22.27 & 0.000 \\
\hline after training & Group $\times$ Generalization of format & $1,702.4$ & 31.68 & 0.000 \\
\hline
\end{tabular}

sively improved their reading performance. Nevertheless, children with dyslexia did so to a lesser extent than controls. Furthermore (and critically for the present analysis), the children with dyslexia failed to transfer learning of a new format to new untrained items, indicating that their improvement during training was entirely due to the learning of specific novel words. As experience with both format and stimulus materials was minimized, the failure of children with dyslexia to attune to a new graphic format cannot be easily attributed to reduced exposure to print and indicates a genuine perceptual disability to learn a new format (despite partially spared ability to learn specific items). Namely, children with dyslexia were unable to build-up an efficient, fast, parallel processing of multiple letters displayed in a new array. We suggest that their performance in this experiment mimics (in a comparatively short time window) their failure to learn to read at school in the standard horizontal array. The reduced ability to cope with zigzag format of children with dyslexia contrasts with the ability of typically developing readers to adapt to this new perceptual condition and transfer learning to new items.

The present findings are in keeping with the idea that VWFA hypo-activation in children with dyslexia ${ }^{16}$ is not a by-product of limited reading experience but indicates selective inability to build up an efficient hierarchy of detectors tuned to larger and more complex word fragments. The VWFA provides a very flexible perceptual task-specific mechanism that allows reading regardless of size, font, cursive or capital letters, and even when atypical features ${ }^{19}$ or other sensory modalities ${ }^{20}$ define the shape of the letters. In good readers, the VWFA ensures tolerance for a certain degree of deviation from the standard horizontal format (small rotation of letters, size etc. ${ }^{4}$ ). The present data gathered in typically developing children, particularly their fast learning and transfer of learning to untrained items, suggests growing tolerance for misalignment of the pre-existing VWFA mechanism and confirms its high plasticity throughout the lifespan (for adult subjects, $\operatorname{se}^{20}$ ). Thus, the VWFA is also able to learn destructive manipulations of the standard (e.g., zigzag format) possibly through plasticity of binding between detectors starting from the bigram stage ${ }^{3}$.

The slower learning rate and absence of transfer suggest that this flexible perceptual task-specific mechanism is poorly developed in children with dyslexia. This is consistent with $\mathrm{fMRI}$ results indicating impaired tuning of VWFA ${ }^{21}$ and focal disruption of functional connectivity between VWFA and left inferior frontal and inferior parietal areas, two major components of the language network ${ }^{22}$. The slow, serial, stiff, reading, likely driven by attentional control of dorsal parietal areas ${ }^{14}$, is used by typically developing readers in the case of degraded stimuli ${ }^{4}$ and, likely, also when the zigzag format was administered before learning (present study). More generally, it is used by all beginner readers when they initially learn to read at school in the standard format. However, children with dyslexia are unable to shift toward a fast parallel mode, and after years of reading experi- ence still show strong dependency on stimulus length, a serial processing cue that has been shown in both languages with regular ${ }^{23,24}$, and irregular orthography ${ }^{25}$, and which is dominant only in the early stages of learning in typically developing readers ${ }^{26}$.

Although both dyslexic and typically developing readers improved their performance in training novel words, the representations of the children with dyslexia were item-specific. In the LCD model ${ }^{3}$, the inability to learn a new format indicates a deficit of the mechanisms that allow perceptual learning through local combination detectors; this deficit could be the base of developmental dyslexia. According to the physiological literature, perceptual learning is mediated by sprouting and pruning of connections, with effects that modify the functional properties of neurons even at the $\mathrm{V} 1$ level ${ }^{27}$. One may speculate that plasticity processes taking place through reading experience in VWFA are less efficient in children with dyslexia and thus unable to shape efficient and flexible connections within the VWFA, and possibly also towards other cortical areas relevant in the reading process.

An alternative explanation of present results may be framed within the attentional deficit hypothesis of dyslexia (for reviews, $\left.\operatorname{se}^{28,29}\right)$. According to this view, the ability to dynamically reallocate attention is reduced in dyslexia ${ }^{30,31}$. Thus, the inability to learn the zigzag format may express the difficulty of isolating the appropriate chunk within the target and shifting the locus of attention as the zigzag word form is parsed. Although the presence of an attentional deficit is debated ${ }^{32,33}$, the role of dorsal attention network in reading is supported by increased parietal activity for words presentation in unusual formats ${ }^{4,11,13,14}$. Furthermore, it has been recently reported that the VWFA shows maximal resting-state correlation with the dorsal attention network ${ }^{34}$. Thus, if an attentional deficit were present in dyslexia, one might expect the VWFA to be inefficient because of a poor input. Within the attentional hypothesis, the total lack of transfer to new untrained items would indicate that spatial attention does not improve through repetition. Possibly, the partial improvement present for trained novel words could be seen as due to the partial functioning of the VWFA.

Overall, the present results indicate that children with dyslexia failed to learn a new graphic format despite partially spared ability to learn specific items. By minimizing experience with both format and stimulus materials we showed that this deficit is not due to reduced exposure to print and indicates a genuine learning perceptual disability. With notable exceptions ${ }^{35}$ research on developmental dyslexia (and more generally on word recognition) has largely relied on single-session experiments and only in the last few years learning designs are starting to be used more extensively (e.g. ${ }^{36}$,). For example, it has been recently found that non-word learning occurred more slowly in college students with a diagnosis of dyslexia than in typical readers (and the length effect persisted for longer periods of time ${ }^{37}$ ). These results are generally consistent with the present findings and 


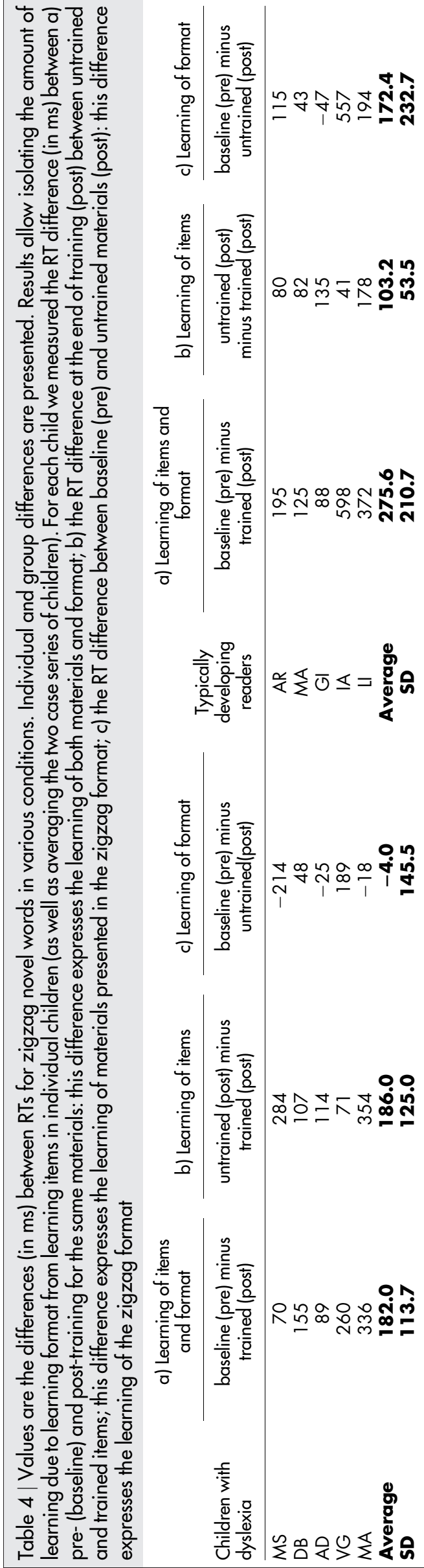

highlight the interest of using learning designs to understand the processes underlying developmental dyslexia.

\section{Methods}

Participants. Five children with dyslexia and five typically developing readers, all Italian native speakers, participated in the training experiment (for a description of these two case series, see Table 1). The school where participants were enrolled collected written informed consent provided by each student's family, as part of an agreement between the school and the Sapienza University of Rome. The ten subjects were selected from a larger group of third- to eight-grade children previously screened for reading level by administering the MT Reading test ${ }^{38,39}$ (approximately, onehundred children per grade were examined). In this standard test, the child reads a text passage aloud with a four-minute time limit; speed (s/syllable) and accuracy (number of errors, adjusted for the amount of text read) are scored. Dyslexia was evaluated based on the ICD-10 criteria (also adopted by the Consensus Conference on learning disabilities of the Italian Superior Institute of Health ${ }^{40}[\mathrm{http}: / / \mathrm{www}$.snlg-iss. it/cms/files/Cc_Disturbi_Apprendimento_sito.pdf]). In particular, children were considered to show dyslexia if they scored at least 2 SDs below the norm for either speed or accuracy on the MT Reading test ${ }^{38}$ and demonstrated normal intelligence (as assessed by Raven's Coloured Matrices ${ }^{41}$; see Table 1). All participants had normal or corrected-to-normal visual acuity. None of them previously underwent any reading or writing intervention.

Five children with dyslexia agreed to participate to the study (out of the 12 identified in $7^{\text {th }}$ and $8^{\text {th }}$ grade). They were administered the first testing session with zigzag novel words (see Stimuli and procedure) to assess their baseline level with the zigzag novel words for both RTs and accuracy. Several younger typically developing children were given the first session to identify those with a closely matched performance in terms of RTs and errors to the five children with dyslexia. An approximate 4 to 1 match was necessary to find the five typically developing children closely matching the performance of the five children with dyslexia. Note that the total number of children was comparatively small. However, dyslexia in regular orthographies is more homogeneous than in irregular orthographies ${ }^{42}$; furthermore, to enhance the reliability of the present data we used a number of training session sufficient to obtain reliable estimates of the learning curves also at an individual level (see below). The individual values of the matched pairs are shown in the baseline columns of Table 2 (top and bottom for RTs and accuracy, respectively).

Stimuli and procedure. Two lists (A and B, see Appendix) of 50 five-letter novel words were derived from two lists of words, which were matched for age of acquisition, frequency (based on CoLFIS database ${ }^{43}$ [http://linguistica.sns.it/CoLFIS/ Home.htm]), bigram frequency and initial phoneme. Non-words were generated from words by changing one or two letters but maintaining the consonant-vocal sequence of the original word. The novel word lists were balanced for bigram frequency and initial phoneme. Novel word list $\mathrm{A}$ was presented in the zigzag format in the first session (baseline) and then during training; novel word list B was used at the end of training to evaluate transfer of format learning.

At the viewing distance of $57 \mathrm{~cm}$, each letter (Courier New, black on a white background) subtended $0.4 \mathrm{deg}$, and the whole stimulus $3.2 \mathrm{deg}$, horizontally. The second and the fourth letters were shifted upward by 0.8 deg to obtain the zigzag format, as in the following example (the novel word "vepra"):

$$
\text { v } \mathrm{e}_{\mathrm{p}}^{\mathrm{r}} \mathrm{a}
$$

Centre-to-centre letter distance was 1.3 deg between adjacent letters on the same line and 1.0 deg between letters in diagonal. Novel words were displayed singly on a PC screen controlled by the DMDX software ${ }^{44}$. Order of presentation followed a new randomized order in each session. The children were instructed to read the novel word aloud as fast and accurately as possible. A voice key connected to the PC recorded vocal RTs at the onset of pronunciation, which triggered the disappearance of the stimulus. No feedback on reading accuracy was given to the children.

The experiment was performed at school, in a quiet room. A total of 23 testing sessions were given. In general, a substantial number of training sessions is necessary to reliably estimate the power functions describing learning (often 10 to $15^{18}$ ). However, we chose to use a few additional sessions, as we were interested in obtaining reliable curves also at an individual level. The first and last sessions served as a pre-training baseline and as post-training evaluation, respectively. In the first session, a practice list with six items was given separately to familiarize the child with the task. The next twenty-two testing sessions (including the last post-training evaluation) were administered at school presenting the same set of novel words (but every time in a different randomized order). To keep the experiment in a manageable time window, two sessions per day were carried out. In the last session, we measured post-training performance with the same list. The generalization effect was evaluated with a list of 50 new novel words (list B) presented in a separate block.

Data analysis. Check-Vocal software ${ }^{45}$ was used for off-line evaluation of audiorecordings to validate RTs and check for errors. Median RTs were calculated only for correct responses.

Learning curves were calculated by interpolation of the experimental points with power functions according to equation (1): 


$$
\mathrm{y}=\mathrm{ax}^{(\mathrm{b})}+\mathrm{c}
$$

where $y$ is the reaction time, $a$ is the difference between initial and asymptotic performance, $x$ is the session number (i.e., the amount of practice), $b$ is the exponent that determines the shape of the function, and $c$ is the asymptote indicating the limit of performance.

Linear mixed effect model analyses. Pre-post RT training effects were analyzed with linear mixed effect model analyses ${ }^{46,47}$. This is a robust analysis that allows controlling for the variability of items and subjects; it prevents the potential lack of power of bysubject and by-item analyses and limits the loss of information due to prior averaging of by-item and by-subject analyses.

Four different analyses were carried out. In each analysis, the dependent variable was the individual child's vocal RT on each item in each experimental condition. Participant (i.e., the "super-subject" made by each dyslexic child and their paired control) and item were entered in each analysis as random factors and group (children with dyslexia vs typically developing readers) as a fixed factor. Furthermore, depending on the objectives of each analysis, the following were entered as fixed factors: $A$ ) training (comparing pre vs post RTs in reading zigzag novel words object of training); $B$ ) session (comparing performance on each of the 23 presentations with the novel words of the trained list); $C$ ) stimulus material (comparing post-training RTs on trained novel words to RTs to "new" untrained novel words); and D) generalization of format (comparing RTs in pre-training to RTs to untrained novel words after training).

1. Shaywitz, S. E. Dyslexia. New Engl. J. Med. 338, 307-12 (1998).

2. Stanovich, K. E. Matthew effects in reading: some consequences of individual differences in the acquisition of literacy. J. Educ. 189, 23-55 (2008/2009).

3. Dehaene, S., Cohen, L., Sigman, M. \& Vinckier, F. The neural code for written words: a proposal. Trends Cogn. Sci. 9, 335-341 (2005).

4. Cohen, L., Dehaene, S., Vinckier, F., Jobert, A. \& Montavont, A. Reading normal and degraded words: contribution of the dorsal and ventral visual pathways. Neuroimage 40, 353-366 (2008).

5. Dehaene, S. et al. Letter binding and invariant recognition of masked words. Psychol. Sci. 15, 307-313 (2004).

6. Vinckier, F. et al. Hierarchical coding of letter strings in the ventral stream: dissecting the inner organization of the visual word-form system. Neuron 55, 143-156 (2007).

7. Legge, G. E., Ahn, S. J., Klitz, T. S. \& Luebker, A. Psychophysics of reading--XVI. The visual span in normal and low vision. Vision Res. 37, 1999-2010 (1997).

8. Koriat, A. \& Norman, J. Reading rotated words. J. Exp. Psychol. Hum. Percept. Perform. 11, 490-508 (1985).

9. Spinelli, D., De Luca, M., Judica, A. \& Zoccolotti, P. Crowding effects on word identification in developmental dyslexia. Cortex 38, 179-200 (2002).

10. Lavidor, M. An examination of the lateralized abstractive/form specific model using MiXeD-CaSe primes. Brain Cogn. 48, 413-417 (2002).

11. Mayall, K., Humphreys, G. W., Mechelli, A., Olson, A. \& Price, C. J. The effects of case mixing on word recognition: evidence from a PET study. J. Cognitive Neurosci. 13, 844-853 (2001).

12. Seymour, P. H. K. \& Macgregor, C. J. Developmental dyslexia: a cognitive experimental analysis of phonological, morphemic, and visual impairments. Cogn. Neuropsychol. 1, 43-82 (1984).

13. Rosazza, C., Cai, Q., Minati, L., Paulignan, Y. \& Nazir, T. A. Early involvement of dorsal and ventral pathways in visual word recognition: an ERP study. Brain Res. 1272, 32-44 (2009).

14. Pammer, K., Hansen, P., Holliday, I. \& Cornelissen, P. Attentional shifting and the role of the dorsal pathway in visual word recognition. Neuropsychologia 44, 2926-2936 (2006).

15. Spinelli, D. et al. Length effect in word naming latencies: role of reading experience and reading deficit. Dev. Neuropsychol. 27, 217-235 (2005).

16. Richlan, F., Kronbichler, M. \& Wimmer, H. Functional abnormalities in the dyslexic brain: a quantitative meta-analysis of neuroimaging studies. Hum. Brain Mapp. 30, 3299-3308 (2009).

17. Newell, A. \& Rosenbloom, P. S. Mechanisms of skill acquisition and the law of practice. Cognitive skills and their acquisition. Anderson, J. R. (ed.) 1-55 (Erlbaum, Hillsdale, NJ, 1981).

18. Logan, G. D. Shapes of reaction-time distributions and shapes of learning curves: a test of the instance theory of automaticity. J. Exp. Psychol.: Learn. Mem. Cogn. 18, 883-914 (1992).

19. Rauschecker, A. M. et al. Visual feature-tolerance in the reading network. Neuron 71, 941-953 (2011).

20. Striem-Amit, E., Cohen, L., Dehaene, S. \& Amedi, A. Reading with sounds: sensory substitution selectively activates the visual word form area in the blind. Neuron 76, 640-652 (2012).

21. van der Mark, S. et al. Children with dyslexia lack multiple specializations along the visual word form (VWF) system. NeuroImage 47, 1940-1949 (2009).

22. van der Mark, S. et al. The left occipitotemporal system in reading: disruption of focal fMRI connectivity to left inferior frontal and inferior parietal language areas in children with dyslexia. NeuroImage 54, 2426-2436 (2011).
23. Spinelli, D. et al. Crowding effects on word identification in developmental dyslexia. Dev. Neuropsychol. 27, 217-235 (2005).

24. De Luca, M., Borrelli, M., Judica, A., Spinelli, D. \& Zoccolotti, P. Reading words and pseudowords: an eye movement study of developmental dyslexia. Brain Lang. 80, 617-626 (2002).

25. Ziegler, J. C., Perry, C., Ma-Wyatt, A., Ladner, D. \& Schulte-Korne, G. Developmental dyslexia in different languages: language-specific or universal? J. Exp. Chi. Psych. 86, 169-193 (2003).

26. Zoccolotti, P. et al. Word length effect in early reading and in developmental dyslexia. Brain Lang. 93, 369-373 (2005).

27. Gilbert, C. D. \& Li, W. Adult visual cortical plasticity. Neuron 75, 250-264 (2012).

28. Valdois, S., Bosse, M. L. \& Tainturier, M. J. The cognitive deficits responsible for developmental dyslexia: review of evidence for a selective visual attentional disorder. Dyslexia 10, 339-363 (2004).

29. Vidyasagar, T. R. \& Pammer, K. Dyslexia: a deficit in visuo-spatial attention, not in phonological processing. Trends Cogn. Sci. 14, 57-63 (2009).

30. Lallier, M., Donnadieu, S., Berger, C. \& Valdois, S. A case study of developmental phonological dyslexia: Is the attentional deficit in the perception of rapid stimuli sequences amodal? Cortex 46, 231-241 (2010).

31. Facoetti, A., Ruffino, M., Peru, A., Paganoni, P. \& Chelazzi, L. Sluggish engagement and disengagement of non-spatial attention in dyslexic children. Cortex 44, 1221-1233 (2008).

32. Ramus, F. Developmental dyslexia: specific phonological deficit or general sensorimotor dysfunction? Curr. Opin. Neurobiol. 13, 212-218 (2003).

33. Ziegler, J. C., Pech-Georgel, C., Dufau, S. \& Grainger, J. Rapid processing of letters, digits and symbols: what purely visual-attentional deficit in developmental dyslexia? Dev. Sci. 13, F8-F14 (2010).

34. Vogel, A. C., Miezin, F. M., Petersen, S. E. \& Schlaggar, B. L. The putative visual word form area is functionally connected to the dorsal attention network. Cereb. Cortex 22, 537-549 (2012).

35. Mayringer, H. \& Wimmer, H. Pseudoname learning by German-speaking children with dyslexia: Evidence for a phonological learning deficit. J. Exp. Child Psychol. 75, 116-133 (2000).

36. Messbauer, V. \& de Jong, P. F. Word, nonword, and visual paired associate learning in Dutch dyslexic children. J. Exp. Child Psychol. 84, 77-96 (2003).

37. Kwok, R. K. \& Ellis, A. W. Visual word learning in adults with dyslexia. Front. Hum. Neurosci. (in press).

38. Cornoldi, C. \& Colpo, G. Nuove prove di lettura MT per la scuola media inferiore, manuale. (O.S. Organizzazioni Speciali, Firenze, 1995).

39. Cornoldi, C. \& Colpo, G. Prove MT per la scuola elementare 2. (O.S. Organizzazioni Speciali, Firenze, 1998).

40. Ministero della Salute e Istituto Superiore di Sanità (2011). Disturbi specifici dell'apprendimento. Consensus Conference, 6-7 December 2010.

41. Pruneti, C. A. et al. Aggiornamento alla standardizzazione italiana del test delle Matrici Progressive Colorate di Raven (CPM) [Update of the Italian standardization of Raven's Coloured Progressive Matrices]. Bollettino di Psicologia Applicata 217, 51-57 (1996).

42. Wimmer, H. \& Schurz, M. Dyslexia in regular orthographies: Manifestation and causation. Dyslexia 16, 283-299 (2010).

43. Bertinetto, P. M. et al. (2005). Corpus e Lessico di Frequenza dell'Italiano Scritto (CoLFIS).

44. Forster, K. I. \& Forster, J. C. DMDX: a windows display program with millisecond accuracy. Behav. Res. Meth. Ins. 35, 116-124 (2003).

45. Protopapas, A. CheckVocal: a program to facilitate checking the accuracy and response time of vocal responses from DMDX. Behav. Res. Meth. 39, 859-862 (2007).

46. Brysbaert, M. "The language-as-fixed-effect fallacy": Some simple SPSS solutions to a complex problem (Version 2.0) (Tech. Rep. Royal Holloway, University of London, 2007).

47. Baayen, R. H., Tweedie, F. J. \& Schreuder, R. The subjects as a simple random effect fallacy: subject variability and morphological family effects in the mental lexicon. Brain Lang. 81, 55-65 (2002).

\section{Acknowledgments}

This work was supported by grants from the Italian Department of Health to the IRCCS Fondazione Santa Lucia, Rome.

\section{Author contributions}

M.P. designed and performed the experiment and analyzed data. M.D.L. designed and implemented the computerized experiment and carried out part of statistical analyses. A.W.E. designed the project and wrote the manuscript. C.V.M. carried out part of statistical analyses, D.S. and P.Z supervised the project and wrote the manuscript.

\section{Additional information}

Supplementary information accompanies this paper at http://www.nature.com/ scientificreports

Competing financial interests: The authors declare no competing financial interests. 
How to cite this article: Pontillo, M. et al. Failure to learn a new spatial format in children with developmental dyslexia. Sci. Rep. 4, 4869; DOI:10.1038/srep04869 (2014).

(c) (i) $\Theta$ This work is licensed under a Creative Commons Attribution-NonCommercialcc) article's Creative Commons license, unless indicated otherwise in the image credit; if the image is not included under the Creative Commons license, users will need to obtain permission from the license holder in order to reproduce the image. To view a copy of this license, visit http://creativecommons.org/licenses/by-nc-nd/3.0/ 